\title{
Outcomes after Sleeve Gastrectomy: Influence of the Calibrating Bougie
}

Macías MS ${ }^{1}$, Alarcón del Agua I ${ }^{1}$, Moreno AB ${ }^{1}$, Marente MDVC ${ }^{1}$, Pereira Cunill $\mathrm{JL}^{2}$, García Luna PP${ }^{2}, \mathbf{R u i z ~ J P}^{1}$ and Morales-Conde $\mathbf{S}^{1}$

${ }^{1}$ Unit of Bariatric Surgery and Innovation in Minimally Invasive Surgery, Spain

${ }^{2}$ Endocrinology Department, HUVROCIO, Seville, Spain

*Corresponding author: María Socas Macias, Unit of Bariatric Surgery and Innovation in Minimally Invasive Surgery, Seville, Spain, Tel: 0034-677469509; E-mail: mariasocasmas@hotmail.com

Rec date: May 5, 2016; Acc Date: July 29, 2016; Pub date: August 4, 2016

Copyright: (C) 2016 Macias MS, et al. This is an open-access article distributed under the terms of the Creative Commons Attribution License, which permits unrestricted use, distribution, and reproduction in any medium, provided the original author and source are credited.

\begin{abstract}
Purpose: To analyze the outcomes of patients in whom we performed a SG depending on the calibrating bougie.

Methods: Retrospective review of patients who underwent SG. Baseline characteristics were analyzed, and outcomes compared between groups. In order to identify the contribution of each predictor in the \%EWL, a linear regression univariate and multivariate model was constructed.
\end{abstract}

Results: 168 LSG were performed (120 women), mean age 42.9 years and BMI 51.1 . Mortality rate of $0.3 \%$ and morbidity rate of $4.7 \%$. No differences in terms of complications between both groups $(5.8 \%$ vs $3.2 \%, p=0.735)$, while $\% E W L$ was statistically superior in the $40 \mathrm{fr}$ group $(67.4 \pm 17.9$ vs $58.1 \pm 15.6$ at 24 months, $p=0.012)$. Age, larger bougie size and arthropathy influenced negatively on the \%EWL. In the multivariate analysis, the introduction of the variable "surgeon" produced a reduction of the effect of the bougie size in weight loss at 24 months (mean difference between groups is $7.5 ; 95 \% \mathrm{Cl}(3.4-18.3) ; \mathrm{p}=0.173)$.

Conclusions: There are differences regarding \%EWL in the medium term favoring the use of a $40 \mathrm{Fr}$ bougie against the $58 \mathrm{Fr}$ one, with no differences in terms of complications. As well as the bougie, the surgeon plays an important role in the \%EWL.

Keywords: Laparoscopic sleeve gastrostomy; Bougie; \%EWL; Complications; Outcomes

Abbreviations: Fr: French; LSG: Laparoscopic Sleeve Gastrectomy; \%EWL: Percentage of Excess Weight Loss; BMI: Body Mass Index; CI: Confidence Interval

\section{Introduction}

Laparoscopic Sleeve Gastrectomy (LSG) has been increasingly performed and has become a common surgical procedure. At the beginning, it was introduced as a first-stage restrictive operation in super obese patients with a body mass index (BMI) greater than 60 $\mathrm{kg} / \mathrm{m} 2$ or in high-risk patients before a more complex definitive procedure [1-4].

Nowadays it has been accepted as a definitive stand-alone procedure due to the excellent results in terms of excess weight loss (\%EWL) and control of comorbidities, with significant 3 and 5-year experiences that justify this recommendation [5-14].

For this reason nowadays the indications for LSG include a wide range of patients, such as those with BMI between 35 and $60 \mathrm{~kg} / \mathrm{m}^{2}$, with or without different medical comorbidities, and is also being proposed for patients with moderate obesity $\left(\mathrm{BMI}<35 \mathrm{~kg} / \mathrm{m}^{2}\right)$ as a surgical technique to be taken into account $[6,7,11]$ as a primary procedure.
It is a simple technique which involves a longitudinal resection of the greater curvature of the stomach around a sizing bougie in order to calibrate the final volume of the gastroplasty [15-20].

And even though in the International Sleeve Gastrectomy Expert Panel Consensus (held in March 2011 in Florida, USA), [21] 87\% of participants thought that the optimal bougie size was between 32 and $36 \mathrm{~F}$ (in order to warranty better weight loss and its maintenance) [22-24], up to now there is no evidence based consensus on the optimal size of the bougie we should use [4]. This is probably due to a greater range of complications described in the smaller bougie sizes [18-20].

Despite the fact that it is probably one of the main steps in the standardization of the technique, as well as probably the key to success for this restrictive procedure, up to now it is still widely discussed if the size of the bougie influences the \%EWL by itself and/or the rate of complications. This is the reason why the objective of our review was to analyze the short and medium term outcome of the patients in whom we performed a LSG, depending on the size of the calibrating bougie used in relation to the percentage of excess weight loss and the rate of complications.

\section{Methods}

We performed a retrospective review (based on a prospective database) of all the patients who underwent LSG at our hospital between 2009 and 2013. All of them were evaluated preoperatively by a multidisciplinary bariatric team. The preoperative study included abdominal ultrasound and upper gastrointestinal series (barium 
Page 2 of 5

swallow) in all the patients; and endoscopy, esophageal manometry and 24-hour esophageal $\mathrm{pH}$ study only in the case of patients under proton-pump inhibitor therapy due to the presence of daily symptoms of esophageal reflux. The indications to perform the LSG were BMI $>50$, age $>55$ years, high surgical risk (multiple medical comorbidities or anticoagulation therapy), risks of adhesions due to previous major surgery or incarcerated ventral hernia and the contraindications were the presence of severe gastroesophageal reflux disease with Esophagitis or a huge hiatal hernia.

The analyzed data included sex, preoperative age, BMI, bougie size, surgeon, short term postoperative morbidity (leaks, abscesses and stenosis) and \%EWL at 6 months and annually. We used two different sizes of bougie according to the preference of the surgeon (40 Fr or 58 Fr). Outcomes were compared between the two bougie sizes ( 40 or 58 $\mathrm{Fr})$ to determine the differences in terms of complication rate and \%EWL. The collected data were analyzed using IBM SPSS Statistics 19.0 (SPSS Inc. Chicago, IL, USA) and included descriptive statistics. Quantitative data were expressed by mean +/- standard deviation, and qualitative data were represented by absolute frequency and percentage. We used the Kolmogorov-Smirnov's test to check the normal distribution and the Student's test and Chi-square test (or Fisher test) for analysis between groups. Pearson's correlation coefficient was conducted for the analysis of the association of quantitative variables and \%EWL and ANOVA for the analysis of the variable "surgeon". To identify the contribution of each predictor for the \%EWL, a linear regression univariate and multivariate model was constructed. To interpret the impact of each significant predictor, the regression coefficient (b) was used. For the multivariate analysis, we selected the variables statistically significant adding the surgeon's factor as a correction element. In all analyses, a two-tailed $p$ value of 0.05 or less was considered statistically significant.

\section{Main steps of our standardized technique}

Dissection begins along the medial greater curvature up to the left crus, dissecting the angle of Hiss completely free. Prior to the gastrectomy the anesthesiologist inserts the 40 or 58 Fr bougie (depending on the surgeon's preference) under laparoscopic vision.

Two graspers are used to place the bougie medially along the lesser curvature. The second assistant retracts the greater curvature toward the patient's left side and the surgeon's left hand begins the sleeve gastrectomy starting $5 \mathrm{~cm}$ proximal to the pylorus (at the level of the "crow's foot"), but symmetrically all the way between the anterior and posterior gastric wall, avoiding the twisting of the staple line, and close enough to allow for the smooth mobilization of the bougie during all the procedure.

This maneuver helps to place the stapler close to the bougie, maintaining the ending volume of the pouch constant. We routinely use buttressing material in all the cartridges to protect the staple line from bleeding (Seamguard Bioabsorbable, Gore-Tex, Flagstaff, AZ).

We complete the sleeve gastrectomy with a total of 5-6 staple firings (two $4.8 \mathrm{~mm}$ and three $3.8 \mathrm{~mm}$ cartridges, $60 \mathrm{~mm}$ length, Echelonflex $^{\oplus}$, Johnson \& Johnson). Next, the anesthesiologist removes the bougie and we check the integrity of the staple line systematically with a methylene blue test. Postoperatively, the patient begins taking clear liquids in the first 24 hours, and is discharged home by the third postoperative day.

\section{Results}

\section{Patients' characteristics}

A total of 168 morbid obese patients (48 men and 120 women) with a mean age of 42.9 years old (18-68) and mean BMI of 51.1 (39.3-66.9) underwent LSG in our Unit for a period of 5 years.

All of them were performed by laparoscopy ( 22 patients by Single Port), with no cases of conversions. And all of these patients underwent LSG as a single-stage operation. A second step being indicated only if needed.

The bougie was specified only in 149 of the 168 patients; in 86 of them a $40 \mathrm{fr}$ bougie was used, and a $58 \mathrm{fr}$ one in 63 patients.

After stratifying patients into groups according to the bougie size, we did not find a significant difference in the baseline characteristics (sex, age, weight, comorbidities (Table 1).

\begin{tabular}{|c|c|c|c|}
\hline & $\begin{array}{l}\text { 40FR GROUP } \\
(n: 86)\end{array}$ & $\begin{array}{ll}58 & \text { FR } \\
\text { GROUP } & \text { (n: } \\
63) & \end{array}$ & $P$ value \\
\hline $\operatorname{Sex}(w)$ & $69.7(60)$ & $73.0(46)$ & 0.666 \\
\hline Preoperative age $(\mathrm{y})$ & $42.6+/-9.0$ & $42.8+/-12.1$ & 0.91 \\
\hline Preoperative weight $(\mathrm{kg})$ & $136.7+/-27.2$ & $141.3+/-19.7$ & \\
\hline Preoperative BMI (kg/m2) & $49.5+/-5.8$ & $52.5+/-4.6$ & 0.001 \\
\hline \multicolumn{4}{|l|}{ Comorbidities \%(n) } \\
\hline Diabetes & $12.8(11)$ & $28.6(18)$ & 0.016 \\
\hline Hypertension & $41.9(36)$ & $55.6(35)$ & 0.098 \\
\hline Dyslipidemia & $19.8(17)$ & $30.2(19)$ & 0.143 \\
\hline OSA & $24.4(21)$ & $28.6(18)$ & 0.569 \\
\hline Arthropathy & $14.0(12)$ & $19.0(12)$ & 0.403 \\
\hline
\end{tabular}

BMI: Body Mass Index. OSA: Obstructive Sleep Apnea. Quantitative data represented by: mean $+/$ - standard deviation, and qualitative data represented by: $\%(n) . P$ value calculated with Chi-square test and T student test.

Table 1: Patients characteristics depending on the size of the bougie used (n: 149) except for the BMI, because one of the main indications of this technique in our unit is the presence of super-obesity (BMI $>50$ ).

\section{Morbidity and mortality}

Average hospital stay was 3.6 days. The mortality rate was $0.3 \%$ (one patient who suffered a leakage at the angle of Hiss with a 40Fr bougie), with a global morbidity rate in the short term of $4.7 \%$ (4 leakages, 2 stenosis in the cisura angularis and 1 abscess), but only $3.3 \%$ of the patients required reoperations to solve their complications $(71 \%$ of the complicated patients: both stenosis, one in the $40 \mathrm{fr}$ and another one in the $58 \mathrm{Fr}$ group, and 3 leakages in the $40 \mathrm{Fr}$ group). Even though the rate of complications was superior in the $40 \mathrm{fr}$ group, these results were not statistically significant (Table 2). 
Citation: Macías MS, Agua Ad I, Moreno AB, Marente MDVC, Pereira Cunill JL, et al. (2016) Outcomes after Sleeve Gastrectomy: Influence of the Calibrating Bougie. Gen Med (Los Angeles) 4: 264. doi:10.4172/2327-5146.1000264

Page 3 of 5

\begin{tabular}{|l|l|l|l|}
\hline & $\begin{array}{l}\text { 40FR GROUP } \\
\text { (n: 86) }\end{array}$ & $\begin{array}{l}\text { 58 FR GROUP } \\
\text { (n:63) }\end{array}$ & P value \\
\hline Global complication rate & $5.8(5)$ & $3.2(2)$ & 0.735 \\
\hline
\end{tabular}

Short term complication rate

\begin{tabular}{|l|l|l|l|}
\hline Leak & $3.5(3)$ & $1.6(1)$ & 0.402 \\
\hline Stenosis/kinking & $1.2(1)$ & $1.6(1)$ & 0.999 \\
\hline Abscess & $1.2(1)$ & $0(0)$ & - \\
\hline Reoperations (\%) & $4.7(4)$ & $1.6(1)$ & 0.5897 \\
\hline
\end{tabular}

Average \%EWL

\begin{tabular}{|l|l|l|l|}
\hline 3 months & $29.2 \pm 11.4(84)$ & $29.0 \pm 9.8(61)$ & 0.93 \\
\hline 6 months & $51.3 \pm 11.7(83)$ & $45.6 \pm 11.0(62)$ & 0.003 \\
\hline 1 year & $65.1 \pm 16.4(79)$ & $56.1 \pm 14.6(60)$ & 0.001 \\
\hline 2 years & $67.4 \pm 17.9(42)$ & $58.1 \pm 15.6(44)$ & 0.012 \\
\hline 3 years & $66.9 \pm 16.5(17)$ & $55.5 \pm 14.7(15)$ & 0.048 \\
\hline
\end{tabular}

$\%$ EWL: percentage of excess weight loss. Qualitative data represented by: \% (n). Quantitative data represented by: mean +/- standard deviation, and (n) represented the number of patients analyzed in each follow up period. $P$ value calculated with Fisher's test.

Table 2: Complication rate and percentage of excess weight loss according to the size of the bougie used (n: 149).

\section{Follow up and \%EWL}

The average follow up was 21 months, with a mean follow-up rate at 3 and 6 months of $97.6 \%$, and at 1,2 and 3 years of $94 \%, 57.7 \%$ and $20 \%$ respectively. The maximum weight loss was reached at 2 years in both groups, with a mean \%EWL of $67 \%$. In relation to the size of the bougie, we analyzed the \%EWL at 3 and 6 months, 1, 2 and 3 years. The profile weight loss in all the patients is represented in (Figure 1).

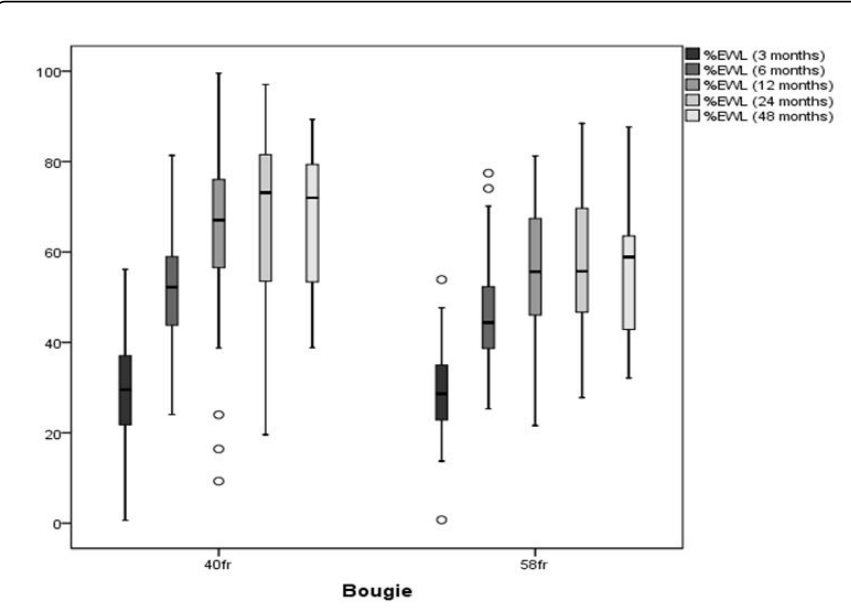

Figure 1: \%EWL profile, including all the patients during the whole follow up period.

(\%EWL: percentage of excess weight loss) and is similar to the profile of the patients who reached a 3 year follow up (17 patients in the $40 \mathrm{fr}$ group and 13 patients in the $58 \mathrm{fr}$ group). These differences were statistically significant from 6 months onwards in favor of the $40 \mathrm{fr}$ bougie, with weight loss maintenance in the medium and long term (Table 2).

\section{Univariate and multivariate analysis}

At 24 months, neither gender nor BMI nor development of complications or intervenient surgeon seem to influence the \%EWL. Only age (Pearson's correlation $(r)=-0.4, p<0.001)$, bougie size (mean difference between groups is 9.3 ; $95 \%$ CI $(2.1-16.5)$; t test $=2.559$, degrees of freedom: $84 ; \mathrm{p}=0.012$ ) and the presence of arthropathy (mean difference between groups is 10.8 ; 95\% CI $(1.9-19.7)$; t test $=2.559$, degrees of freedom: $84 ; \mathrm{p}=0.018$ ), were factors that influenced negatively on weight loss. Even though the surgeon was not shown as a determinant factor in the univariate analysis, in the multivariate analysis the introduction of the variable "surgeon" produced a reduction of the effect of the bougie size in weight loss at 24 months (mean difference between groups is 7.5 ; 95\% CI $(3.4-18.3)$; $\mathrm{p}=0.173$ ) (Table 3).

\begin{tabular}{|l|l|l|l|l|l|l|}
\hline \multirow{2}{*}{} & \multicolumn{4}{l}{ UNIVARIATE } & \multicolumn{4}{l|}{ MULTIVARIATE } \\
\cline { 2 - 7 } & B & CI 95\% (B) & $p$ & B & CI 95\% (B) & $p$ \\
\hline Age (years) & -0.7 & $(-1.0 ;-0.4)$ & $<0.001$ & -0.6 & $(-1.0 ;-0.3)$ & 0.001 \\
\hline $\begin{array}{l}\text { Sex (male/ } \\
\text { female) }\end{array}$ & -3.8 & $(-12.0 ; 4.4)$ & 0.357 & & & \\
\hline $\begin{array}{l}\text { BMI } \\
\left.\text { (kg/m }{ }^{2}\right)\end{array}$ & -0.2 & $(-1.0 ; 0.5)$ & 0.535 & & & \\
\hline $\begin{array}{l}\text { Bougie } \\
(58 / 40 f r)\end{array}$ & -9.3 & $(-16.5 ;-2.1)$ & 0.012 & -7.5 & $(-18.3 ; 3.4)$ & 0.173 \\
\hline
\end{tabular}

Main surgeon (REF. Surgeon (1))

\begin{tabular}{|l|l|l|l|l|l|l|}
\hline Surgeon (2) & 3.4 & $(-6.8 ; 13.6)$ & 0.507 & 0.2 & $(-9.1 ; 9.6)$ & 0.963 \\
\hline Surgeon (3) & -5 & $(-15.4 ; 5.4)$ & 0.34 & $-130 \%$ & $(-14.6 ; 12.1)$ & 0.847 \\
\hline Surgeon (4) & -3.6 & $(-15.1 ; 7.9)$ & 0.535 & 0.8 & $(-11.9 ; 13.6)$ & 0.896 \\
\hline $\begin{array}{l}\text { Morbidity } \\
\text { (yes/no) }\end{array}$ & -2.1 & $(-19.8 ; 15.6$ & 0.816 & & & 0.339 \\
\hline $\begin{array}{l}\text { Arthropathy } \\
\text { (yes/no) }\end{array}$ & -10.8 & $(-19.7 ;-1.9)$ & 0.018 & -4.4 & $(-13.4 ; 4.7)$ & \\
\hline
\end{tabular}

BMI: Body Mass Index; REF. Surgeon (1): was the reference category for the variable main surgeon in the linear regression model; Morbidity: includes both presence of leaks and stenosis.(Data analysed by using linear regression were the ones at 24 months)

Table 3: Linear regression - univariate and multivariate analysis (n: 86).

\section{Discussion}

LSG has a risk profile and effectiveness that are placed between laparoscopic gastric banding and Roux-en-Y gastric bypass [21-25]; this is the reason why it is recommended in complex patients such as those with greater BMI or a higher rate of comorbidities in order to minimize the complication rate $[21,22]$. 
But LSG is not complication-free at all and one of the major postoperative complication is still the staple line leak, which occurs in approximately $2.2-2.4 \%$ of patients [11-26]. There are various factors which influence the risk of leak. One suggested risk factor is the size of the bougie used in the procedure. In the Second International Consensus Summit for Sleeve Gastrectomy [15] there was a trend toward using a $36 \mathrm{Fr}$ bougie, because it seemed that the use of a smaller bougie size could be associated with a long term increase in weight loss [27-29]. However later on, in 2010 it was suggested by Gagner M et al. that these small bougies were related to an increase in the rate of leakage [18]. In fact, in the systematic analysis performed by Aurora $\mathrm{AR}$ et al. [26], the use of bougies with diameter $>40 \mathrm{Fr}$ resulted in decreased instances of staple line leakage compared to the use of bougies with diameter $<40 \mathrm{Fr}$. In this paper the use of a $40 \mathrm{Fr}$ or a larger bougie was associated to a leak rate of $0.6 \%$, while smaller bougie sizes were associated to a leak rate of $2.8 \%$. Later on, Parik et al. published a systematic review showing that utilizing bougie $\geq 40 \mathrm{Fr}$ might decrease the rate of leakage without having an impact on \%EWL up to 3 years [19].

In our series of patients we have suffered an average leak rate of $2.7 \%$, and we have used $40 \mathrm{Fr}$ and $58 \mathrm{Fr}$ bougies, both considered as large sizes in the literature. And despite the fact that we have used "large" bougies, we have still suffered a rate of leakage higher in the 40 Fr group (3.5\%), than in the 58 Fr group (1.6\%), although these differences were not found statistically significant. These results are similar to the ones published by Atkins ER et al. [30] in their retrospective review, showing a similar rate of complications in the case of $40 \mathrm{Fr}$ and $50 \mathrm{Fr}$ bougies (2.7\% vs $1.9 \%$ leak rate). And according to their results regarding weight loss, and contrary to what has been published before by Parik et al. [19] the group of patients in whom we used a smaller sizing bougie ( $40 \mathrm{Fr}$ ) showed better results of weight loss compared to those patients who underwent LSG using a $58 \mathrm{Fr}$ bougie ( $67.4 \pm 17.9$ vs $58.1 \pm 15.6 \% \mathrm{EWL}$ at 24 months), and these differences were evident and statistically significant from 6 months onwards.

According to the review recently published by Yuval et al. [20], and contrary to what has been said before, the recommendation to use the smallest bougie as possible should be avoided from now on, because the risks of leak may outweigh the benefits $(0.9 \%$ leaks with bougies $\geq 40 \mathrm{Fr}$ vs $2.9 \%$ leaks with bougies $<40 \mathrm{Fr}, \mathrm{p}<0.05$ ). They conclude that larger size bougies are associated with a significant decrease in the incidence of leak with no change in weight loss (69.2\% of EWL $\geq 40 \mathrm{Fr}$ bougie and $60.7 \%$ of EWL when smaller bougies were used, $\mathrm{p}=0.29$ ). In our group we have followed this recommendation, but in our series of patients in whom we have used "large" bougies, we have found an average higher incidence of leaks (2.7\%) close to the incidence described for the $<40$ Fr bougies. Perhaps at this point "other factors" related to the cause of the leak such as the higher BMI [19] could have played its part. In our analysis the mean BMI in the 40 Fr group was $49.5+/-5.8$, and in the 58 Fr group $52.5+/-4.6$, higher than the ones included in Yuval's review (Bougie $<40 \mathrm{Fr}$ an average BMI of 46, and Bougie $>40 \mathrm{Fr}$ an average BMI 43). On the other hand, regarding the $\% \mathrm{EWL}$, we have found differences in the univariate analysis favoring the use of the $40 \mathrm{Fr}$ size bougie instead of the $58 \mathrm{Fr}$ one $(67.4+/-17.9$ vs $58.1+/-15.6 \% \mathrm{EWL}$ at 24 months, $\mathrm{p}<0.05)$. These differences were maintained statistically significant until the third postoperative year (Tables 2 and 3 ).

In the univariate analysis the age and the presence of arthropathy were also factors which negatively influenced weight loss in our patients, probably related to the reduction of the physical activity in the postoperative period whereas in the multivariate analysis, the effect of the bougie was eliminated when we introduced the variable "surgeon". Bearing in mind, that all of the surgeons in the team were performing the same standardized technique we described before, this could mean that in weight loss the size of the bougie is as important as how much the surgeon stays close to it to create the pouch (Table 3).

Even considering the limitations of this retrospective review, we can conclude that when following the same standardized technique during LSG, there are statistically significant differences regarding the \%EWL in the medium term that favor the use of a $40 \mathrm{Fr}$ bougie against the 58 Fr one, with no differences in terms of complications. There are other factors that negatively influence weight loss such as age and the presence of arthropathy. Besides the bougie, the surgeon plays an important role in the \%EWL. Further studies are needed before a definitive decision on the ideal bougie size is made.

\section{References}

1. Gagner M, Gumbs AA, Milone L, Yung E, Goldenberg L et al.(2008) Laparoscopic sleeve gastrectomy for the super-super obese (Body Mass Index $>69 \mathrm{~kg} / \mathrm{m} 2$. Surg Today. 5: 399-403.

2. Baltasar A, Serra C, Perez N, Bou R, Bengochea M et al. (2005) Laparoscopic sleeve gastrectomy: a multi-purpose bariatric operation. Obes Surg 15: 1124-1128.

3. Deitel M, Crosby RD, Gagner M (2008) The first international consensus summit for sleeve gastrectomy (SG). Obes Surg 18: 487-496.

4. Cottam D, Qureshi FG, Mattar SG, Sharma S, Holover S et al.(2006) Laparoscopic sleeve gastrectomy as an initial weight loss procedure for high-risk patients with morbid obesity. Surg Endosc. 20: 859-863.

5. Braghetto I, Csendes A, Lanzarini E, Papapietro K, Cárcamo C et al. (2012) Is Laparoscopic Sleeve Gastrectomy an Acceptable Primary Bariatric Procedure in Obese Patients? Early and 5-Year Postoperative Results. Surg Laparosc Endosc Percutan Tech 22: 479-486.

6. Boza C, Salinas J, Salgado N, Pérez G, Raddatz A et al.(2012) Laparoscopic sleeve gastrectomy as a stand-alone procedure for morbid obesity: report of 1,000 cases and 3-year follow-up. Obes Surg. 22: 866-871.

7. D'Hondt M, Vanneste S, Pottel H, Devriendt D, Van Rooy F et al. (2011) Laparoscopic sleeve gastrectomy as a single stage procedure for the treatment of morbid obesity and the resulting quality of life, resolution of comorbidities, food tolerance, and 6 year weight loss. Surg Endosc 25: 2498-2504.

8. Todkar JS, Shah SS, Shah PS, Gangwani J (2010) Long-term effects of laparoscopic sleeve gastrectomy in morbidly obese subjects with type 2 diabetes mellitus. Surg Obes Relat Dis. 6: 142-145.

9. Fischer L, Hildebrandt C, Bruckner T, Kenngott H, Linke GR et al.(2012) Excessive Weight Loss after Sleeve Gastrectomy: A Systematic Review. Obes Surg. 22: 721-731.

10. Vidal P, Ramón JM, Goday A, Benaiges D, Trillo L et al. (2013) Laparoscopic Gastric Bypass Versus Laparoscopic Sleeve Gastrectomy as a Definitive Surgical Procedure for Morbid Obesity. Mid-Term Results. Obes Surg 23: 292-299.

11. Brethauer SA, Hammel JP, Schauer PR (2009) Systematic review of sleeve gastrectomy as a staging and primary bariatric operation. Surg Obes Relat Dis 5: 469-475.

12. Vidal J, Ibarzabal A, Romero F, Delgado S, Momblán D et al.(2008) Type 2 diabetes mellitus and the metabolic syndrome following sleeve gastrectomy in severely obese subjects. Obes Surg 18: 1077-1082.

13. Noun R, Chakhtoura G, Nasr M, Skaff J, Choucair N et al.(2012) Laparoscopic Sleeve Gastrectomy for Mildly Obese Patients. (Body Mass Index of $30<35 \mathrm{~kg} / \mathrm{m} 2$ ): Operative Outcome and Short-TermResults. J Obe 2012:813650. 
Citation: Macías MS, Agua Ad I, Moreno AB, Marente MDVC, Pereira Cunill JL, et al. (2016) Outcomes after Sleeve Gastrectomy: Influence of the Calibrating Bougie. Gen Med (Los Angeles) 4: 264. doi:10.4172/2327-5146.1000264

Page 5 of 5

14. Kehagias I, Karamanakos SN, Argentou M, Kalfarentzos F (2011) Randomized clinical trial of laparoscopic Roux-en-Y gastric bypass versus laparoscopic sleeve gastrectomy for the management of patients with BMI $<50 \mathrm{~kg} / \mathrm{m} 2$. Obes Surg 21: 1650-1656.

15. Gagner M, Deitel M, Kalberer TL, Erickson AL, Crosby RD (2009) The Second International Consensus Summit for Sleeve Gastrectomy. Surg Obes Relat Dis. 5: 476-485.

16. Rosenthal RJ, Diaz AA, Arvidsson D, Baker RS, Basso N et al.(2012) International Sleeve Gastrectomy Expert Panel Consensus Statement: best practice guidelines based on experience of 12,000 cases. Surg Obes Relat Dis 8: 8-19.

17. Gagner M, Deitel M, Erickson AL, Crosby RD (2013) Survey on laparoscopic sleeve gastrectomy at the Fourth International Consensus Summit on Sleeve Gastrectomy. Obes Surg 23: 2013-2017.

18. Gagner M (2010) Leaks After Sleeve Gastrectomy Are Associated With Smaller Bougies Prevention and Treatment Strategies. Surg Laparosc Endosc Percutan Tech 20: 166-169.

19. Parikh M, Issa R, McCrillis A, Saunders JK, Ude-Welcome A et al.(2013) Surgical Strategies That May Decrease Leak After Laparoscopic Sleeve Gastrectomy. A Systematic Review and Meta-Analysis of 9991 Cases. Ann Surg 257: 231-237.

20. Yuval JB, Mintz Y, Cohen MJ, Rivkind AI, Elazary R (2013) The Effects of Bougie Caliber on Leaks and Excess Weight Loss Following Laparoscopic Sleeve Gastrectomy. Is there an Ideal Bougie Size? Obes Surg. 23:1685-1691.

21. Trastulli S, Desiderio J, Guarino S, Cirocchi R, Vittorio Scalercio et al. (2013) Review article: Laparoscopic sleeve gastrectomy compared with other bariatric surgical procedures: a systematic review of randomized trials. Surg Obes Relat Dis 9: 816-829.

22. Carlin AM, Birkmeyer JD, Share D, Birkmeyer NJO (2013) The Comparative Effectiveness of Sleeve Gastrectomy, Gastric Bypass, and
Adjustable Gastric Banding Procedures for the Treatment of Morbid Obesity. Ann Surg 257: 791-797.

23. Franco JVA, Ruiz PA, Palermo M, Gagner M (2011) A Review of Studies Comparing Three Laparoscopic Procedures in Bariatric Surgery: Sleeve Gastrectomy, Roux-en-Y Gastric Bypass and Adjustable Gastric Banding. Obes Surg 21: 1458-1468.

24. Hutter MM, Schirmer BD, Jones DB, Ko Clifford Y, Cohen Mark E et al. (2011) First report from the American College of Surgeons Bariatric Surgery Center Network:laparoscopic sleeve gastrectomy has morbidity and effectiveness positioned between the band and the bypass. Ann Surg 254: 420-422.

25. Chang SH, Stoll CRT, Song J, Varela JE, Eagon CJ (2012) The effectiveness and risks of bariatric surgery. An up-to-dated systematic review and meta-analisis 2003 and 2012. JAMA 149: 275-287.

26. Aurora AR, Khaitan L, Saber AA (2012) Sleeve gastrectomy and the risk of leak: a systematic analysis of 4888 patients. Surg Endosc 26:1509-1515.

27. Al Daqal SM, Al-Amoodi MS (2013) Effect of Bougie Size and Level of Gastric Resection on Weight Loss Post Laparoscopic Sleeve Gastrectomy. J Obes Weight Loss Ther 3:6.

28. Weiner RA, Weiner S, Pomhoff I, Jacobi C, Makarewicz W et al. (2007) Laparoscopic Sleeve Gastrectomy - Influence of Sleeve Size and Resected Gastric Volume. Obes Surg 17: 1297-305.

29. Bellanger DE, Greenway FL (2011) Laparoscopic sleeve gastrectomy, 529 cases without a leak: short-term results and technical considerations. Obes Surg 21: $146-145$.

30. Atkins ER, Preen DB, Jarman C, Cohen LD (2012) Improved Obesity Reduction and Co-morbidity Resolution in Patients Treated with 40French Bougie Versus 50-French Bougie Four Years after Laparoscopic Sleeve Gastrectomy. Analysis of 294 Patients. Obes Surg 22: 97-104. 